\title{
Dietary Supplements and Sports Performance: Amino Acids
}

\author{
Melvin Williams \\ Exercise Science, Sport, Physical Education, and Recreation, Old Dominion University, Norfolk, VA. \\ Address correspondence to profrunner@aol.com
}

Received October 9, 2005/Accepted November 4, 2005

\begin{abstract}
This is the third in a series of six articles to discuss the major classes of dietary supplements (vitamins; minerals; amino acids; herbs or botanicals; metabolites, constituents/extracts, or combinations). The major focus is on efficacy of such dietary supplements to enhance exercise or sport performance. Journal of the International Society of Sports Nutrition. 2(2):63-67, 2005
\end{abstract}

Key Words: ergogenic aids, sports supplements, protein

\section{DIETARY PROTEIN AND PROTEIN SUPPLEMENTS}

Protein is one of the most popular dietary supplements marketed to athletes and physicallyactive individuals. Protein supplements have been recommended to athletes to enhance nitrogen retention and increase muscle mass, to prevent protein catabolism during prolonged exercise, to promote muscle glycogen resynthesis following exercise, and to prevent sports anemia by promoting an increased synthesis of hemoglobin, myoglobin, oxidative enzymes, and mitochondria during aerobic training. However, whether or not athletes need more protein is currently debatable. Based on available research, the American College of Sports Medicine, the American Dietetic Association, and the Dietitians of Canada, in their recent joint position stand on nutrition and athletic performance ${ }^{1}$, concluded that protein requirements are higher in very active individuals and suggested that resistance athletes need 1.6-1.7 g protein $/ \mathrm{kg}$ body weight while endurance athletes need approximately $1.2-1.4 \mathrm{~g}$ protein $/ \mathrm{kg}$, values that are about $150-200$ percent of the current United States Recommended Dietary Allowances (RDA). Conversely, in its recent presentation of the Dietary Reference Intakes (DRI) for protein, the National Academy of Sciences ${ }^{2}$ concluded that in view of the lack of compelling evidence to the contrary, no additional dietary protein is suggested for healthy adults undertaking resistance or endurance exercise. Sports nutrition scientists do note that even if athletes need more protein, the recommended amounts are compatible with the current Acceptable Macronutrient Dietary Recommendations (10-35 percent of energy from protein) and may be easily obtained from natural foods in the diet ${ }^{3,4}$. In general, protein supplements are not necessary ${ }^{5,6}$.

However, dietary protein is composed of 20 different amino acids which, if ingested individually, have been theorized to possess ergogenic potential and have been marketed as sports supplements to physically active individuals. Indeed, amino acids are among the top five most popular sports supplements 7.

\section{AMINO ACIDS: ERGOGENIC THEORY}

Amino acids are theorized to enhance performance in a variety of ways, such as increasing the secretion of anabolic hormones, modifying fuel use during exercise, preventing adverse effects of overtraining, and preventing mental fatigue. The following discussion highlights research regarding the ergogenic effects of individual amino acids, various combinations of amino acids, and several special protein dietary supplements.

Tryptophan. Tryptophan (TRYP), is a precursor for serotonin, a brain neurotransmitter theorized to suppress pain. Free tryptophan (fTRYP) enters the brain cells to form serotonin. Thus, tryptophan supplementation has been used to increase serotonin production in attempts to increase tolerance to pain during intense exercise. One study reported significant improvements in time to exhaustion at 80 
percent of maximal oxygen uptake, accompanied by significant reductions in the rating of perceived exertion ${ }^{8}$. However, research with a more appropriate experimental design did not replicate these findings ${ }^{9}$. Moreover, other investigators reported no effect of TRYP supplementation on aerobic endurance performance at 70-75 percent of maximal oxygen uptake ${ }^{6}$. Tryptophan does not appear to be an effective ergogenic ${ }^{10}$.

Branched chain amino acids (BCAA). Some investigators believe that increased levels of serotonin may cause fatigue ${ }^{11}$. During prolonged aerobic endurance exercise, muscle glycogen may become depleted and the muscle may increase its reliance on BCAA for fuel, decreasing the plasma BCAA:fTRYP ratio. Because BCAA compete with fTRYP for entry into the brain, a low BCAA:fTRYP ratio would facilitate the entry of fTRYP to the brain and the formation of serotonin. Hypothetically, BCAA supplementation may delay central nervous system fatigue and enhance performance in prolonged aerobic endurance events by increasing the BCAA:fTRYP ratio and mitigating the formation of serotonin.

BCAA supplementation has been studied for its effects on various types of exercise performance, including ratings of perceived exertion (RPE) during exercise and mental performance following exercise. In general, the findings are equivocal, as are the conclusions from several recent reviews. One investigator concluded that BCAA supplementation reduces RPE and mental fatigue during prolonged exercise and improves cognitive performance after exercise, and also suggests that in some situations BCAA supplementation may improve physical performance, such as during exercise in the heat or in actual competitive races where central fatigue may be more pronounced than in laboratory experiments ${ }^{12}$. However, other reviewers conclude that most studies show no effects of BCAA supplementation on performance, such as prevention of fatigue during prolonged exercise ${ }^{13,14}$ two recent studies support these conclusions. Watson and others ${ }^{15}$ reported no beneficial effects of BCAA supplementation, consumed before and during prolonged cycling to exhaustion at 50 percent $\mathrm{VO}_{2} \max$ in the heat, on performance time, heart rate, and core or skin temperature. Cheuvront and others ${ }^{16}$ reported similar findings with subjects exercising in the heat, noting no significant effect of BCAA supplementation on time-trial performance, cognitive performance, mood, perceived exertion, or perceived thermal comfort. Although current research does not support an ergogenic effect of BCAA supplementation, most investigators recommend additional research.

Glutamine. Glutamine may be theorized to be ergogenic in various ways ${ }^{6}$. It is an important fuel for some cells of the immune system, such as lymphocytes and macrophages, which may be decreased with prolonged intense exercise, such as that related to overtraining. Glutamine may also promote muscle glycogen synthesis, and has been studied for potential enhancement of muscular strength.

Several investigators theorize that athletes who overtrain may experience decreased plasma glutamine levels, which may impair functions of the immune system and predispose the athlete to various illnesses ${ }^{17,18}$. Illness may impair training and eventual performance. Research findings are equivocal, with some studies reporting lower incidence rates of infection among athletes who consumed a glutamine-supplement drink following intense training ${ }^{19}$. However, others reported that although glutamine supplementation helped maintain plasma glutamine levels following intense exercise, it had no effect on various tests of the immune response ${ }^{20}$. Recent reviews indicated that there is little support from controlled studies to recommend glutamine ingestion for enhanced immune function ${ }^{14,21}$.

Although glutamine may simulate muscle glycogen synthesis, reviewers recently concluded that there is no advantage over ingestion of adequate carbohydrate alone ${ }^{14}$. Moreover, several recent studies indicate that neither short-term nor long-term glutamine supplementation has an ergogenic effect on muscle mass or strength performance. Glutamine supplementation one hour prior to testing had no effect on resistance exercise to fatigue, nor did six weeks of glutamine supplementation during resistance training increase lean muscle mass or strength more so than the placebo treatment ${ }^{22,23}$.

Aspartates. Potassium and magnesium aspartates are salts of aspartic acid, an amino acid. They have been used as ergogenics, possibly by enhancing fatty acid metabolism and sparing muscle glycogen utilization or by mitigating the accumulation of ammonia during exercise. The effect of aspartate supplementation on physical performance is equivocal, but about 50 percent of the available studies have indicated enhanced performance in exercise tests of aerobic endurance ${ }^{6}$. Additional research is needed to study the potential ergogenicity and underlying mechanisms of aspartate salt supplementation.. 
Arginine. Arginine supplementation may be theorized to be ergogenic because it is a substrate for nitric oxide (NO) synthesis, a potent endogenous vasodilator that may benefit blood flow and endurance capacity. Several studies involving patients with peripheral arterial disease or clinical symptoms of stable angina pectoris have shown improved exercise capacity with arginine supplementation ${ }^{24,25}$. However, research involving the independent effect of arginine supplementation on the aerobic endurance capacity of healthy athletes has not been uncovered ${ }^{6}$.

Ornithine, lysine and arginine. Ornithine, lysine and arginine have been used in attempts to increase human growth hormone (HGH) production, the theory being to increase lean muscle mass and strength. However, although limited data are available, a number of well-controlled studies, several with experienced weight lifters, reported no increases in $\mathrm{HGH}$ levels or various measures of muscular strength or power ${ }^{26-28}$.

Chromiak and Antonio ${ }^{29}$ reviewed the scientific studies on growth-hormone releasing amino acids (ornithine, lysine and arginine) and indicated that oral doses that are great enough to induce significant growth hormone release are likely to cause gastrointestinal discomfort. Moreover, they reported that no studies found that pre-exercise oral amino acid supplementation augments growth hormone release. They also concluded that no appropriately conducted scientific studies found that oral supplementation with such amino acids before strength training increases muscle mass and strength to a greater extent than strength training alone. They do not recommend the use of specific amino acids to stimulate growth hormone release.

Tyrosine. Tyrosine is a precursor for the catecholamine hormones and neurotransmitters, specifically epinephrine, norepinephrine, and dopamine. Some have suggested that inadequate production of these hormones or transmitters could compromise optimal physical performance. Thus, as a precursor for the formation of these hormones and neurotransmitters, tyrosine has been suggested to be ergogenic. However, in a well-designed placebocontrolled, crossover study, Sutton ${ }^{30}$ and others found that tyrosine supplementation (150 milligrams/kilogram body weight) consumed 30 minutes prior to taking a series of physical performance tests significantly increased plasma tyrosine levels, but had no significant ergogenic effects on aerobic endurance, anaerobic power, or muscle strength.
Taurine. Taurine is a non-essential sulfur-containing amino acid, but it lacks a genetic codon to be incorporated into proteins or enzymes. Nevertheless, it plays a role in several metabolic processes, such as heart contraction and antioxidant activity. Taurine is an ingredient in several so-called energy drinks, such as Red Bull.

Baum and Weiss ${ }^{31}$ reported that Red Bull, which contains taurine and caffeine, as compared to a similar drink without taurine, favorably influence cardiac parameters, mainly an increased stroke volume, during recovery after exercise; however, physical performance was not tested. However, Zhang and others ${ }^{32}$ reported that 7 days of taurine supplementation induced significant increases in $\mathrm{VO}_{2}$ max and cycle ergometer exercise time to exhaustion; the ergogenic effects were attributed to taurine's antioxidant activity and protection of cellular properties.

Amino Acid Cocktails. Providing an ample supply of essential amino acids to the muscle within 1-3 hours before or following exercise may help to further muscle protein synthesis. Gibala ${ }^{33}$ indicated that consumption of a drink containing about 0.1 gram of essential amino acids per kilogram of body weight (7 grams for a 70-kilogram athlete) during the first few hours of recovery from heavy resistance exercise will produce a transient, net positive increase in muscle protein balance. Gibala also noted that it is uncertain if ingesting amino acids, either alone or combined with carbohydrate, immediately before exercise or during recovery further enhances the rate of muscle protein buildup during recovery. Some investigators have suggested that it may be helpful to consume, throughout the day, multiple small meals having adequate protein. Gibala indicates that although these strategies will promote a net "anabolic" environment in the body, it remains to be determined if the acute effects of supplementation eventually lead to greater gains in muscle mass following habitual training. Others also note that small amounts of amino acids, combined with carbohydrates, can transiently increase muscle protein anabolism, but it has yet to be determined if these transient responses result in an appreciable increase in muscle mass over a prolonged training period ${ }^{42,34}$.

Overall, given these findings, consuming a small amount of protein and carbohydrate, either as a protein/carbohydrate energy drink or whole foods, before or after exercise training may be prudent behavior for many athletes. 


\section{WHEY PROTEIN AND COLOSTRUM}

Whey and colostrum are two forms of protein that are theorized to be ergogenic. Whey proteins are extracted from the liquid whey that is produced during the manufacture of cheese or casein, while colostrum is the first milk secreted by cows. Both are rich sources of protein, vitamins and minerals, but may contain various biologically active components, including growth factors ${ }^{35,36}$. Although no mechanism has been identified, one theory involves increased levels of serum insulin-like growth factor (IGF-1), which could be anabolic. However, research with colostrum supplementation shows no effect on blood IGF-I or IGF binding protein levels ${ }^{37}$.

Research regarding the ergogenic effect of whey protein and colostrum supplementation is very limited ${ }^{6}$. In general, research findings are equivocal. For example, one study evaluated the effect of colostrum supplementation on four tests of exercise performance and reported a significant effect on 50meter sprint time, but no effect on vertical jump, shuttle sprinting to exhaustion, or 300-meter sprint time ${ }^{38}$. Brinkworth and others ${ }^{39}$ reported that bovine colostrum supplementation, as compared to whey protein, during 8 weeks of resistance training group increased arm circumference and cross-sectional area, but the increase was due principally to a greater increase in skin and subcutaneous fat. Tipton and others ${ }^{40}$ reported that acute ingestion of both whey protein and casein after exercise resulted in similar increases in muscle protein net balance, resulting in net muscle protein synthesis despite different patterns of blood amino acid responses. Although the results of these studies were suggestive of ergogenic effects, they should be regarded as preliminary and more research is merited.

\section{SAFETY, LEGALITY}

Consumption of high-protein diets $(2.8 \mathrm{~g}$ protein $/ \mathrm{kg}$ or less) by well-trained athletes does not appear to impair renal function, as indicated by various measures of renal function ${ }^{41}$. However, certain individuals should be concerned with the protein content in their diet, such as those with diabetes mellitus predisposed to kidney disease, and those prone to kidney stones ${ }^{6}$. Most amino acid supplements are safe in recommended dosages, but may interfere with protein metabolism if consumed in excess. Use of amino acid supplements is not prohibited by the World Anti-Doping Agency (WADA).

\section{REFERENCES}

1. American Dietetic Association, Dietitians of Canada. American College of Sports Medicine. Nutrition and athletic performance. Journal of the American Dietetic Association 100:1543-46, 2000.

2. National Academy of Sciences. Dietary Reference Intakes for Energy, Carbohydrates, Fiber, Fat, Protein and Amino Acids. Washington, DC: National Academies Press, 2002

3. Lambert, C., et al. Macronutrient considerations for the sport of bodybuilding. Sports Medicine 34:317-27, 2004.

4. Tipton, K., et al. Ingestion of casein and whey proteins result in muscle anabolism after Resistance exercise. Medicine \& Science in Sports \& Exercise 36:2073-81, 2004.

5. Phillips, S. Protein requirements and supplementation in strength sports. Nutrition 20:689-95, 2004.

6. Williams, MH Nutrition for Health, Fitness \& Sports. Boston: McGraw-Hill, 2005.

7. Lawrence, M., and Kirby, D. Nutrition and sports supplements: Fact or fiction. Journal of Clinical Gastroenterology 35: 299-306, 2002.

8. Segura, R., and Ventura, J. Effect of L-tryptophan supplementation on exercise performance. International Journal of Sports Medicine. 9: 301-5, 1988.

9. Stensrud, T., et al. L-tryptophan supplementation does not improve running performance. International Journal of Sports Medicine 13: 481-85, 1992.

10. Williams, M. H. Facts and fallacies of purported ergogenic amino acid supplements. Clinics in Sports Medicine18: 633-49, 1999.

11. Newsholme, E., et al. Physical and mental fatigue: Metabolic mechanisms and importance of plasma amino acids. British Medical Bulletin, 48: 477-95, 1992.

12. Blomstrand, E. Amino acids and central fatigue. Amino Acids 20: 25-34, 2001.

13. Davis, J. M., et al. 2000. Serotonin and central nervous system fatigue: Nutritional considerations. American Journal of Clinical Nutrition 72: 573S-8S, 2000

14. Hargreaves, M., and Snow, R. Amino acids and endurance exercise. International Journal of Sport Nutrition and Exercise Metabolism 11:133-45, 2001.

15. Watson, $\mathrm{P}$., et al. The effect of acute branched-chain amino acid supplementation on prolonged exercise capacity in a warm environment. European Journal of Applied Physiology 93:306-14, 2004.

16. Cheuvront, S., et al. Branched-chain amino acid supplementation and human performance when hypohydrated in the heat. Journal of Applied Physiology 97:1275-82, 2004.

17. Budgett, R., et al. The overtraining syndrome. In Harries, M., et al. Oxford Textbook of Sports Medicine Oxford: Oxford University Press, 1998.

18. Castell, L. Glutamine supplementation vitro and in vivo, in exercise and in immunodepression. Sports Medicine 33:323-45, 2003. 
19. Castell, L., et al. Does glutamine have a role in reducing infections in athletes. European Journal of Applied Physiology 73: 488490, 1996.

20. Rohde, T., et al. Effect of glutamine supplementation on changes in the immune system induced by repeated exercise. Medicine and Science in Sports and Exercise 30: 856-862, 1998.

21. Nieman, D. Exercise immunology: Nutrition countermeasures. Canadian Journal of Applied Physiology 26:S45-55, 2001.

22. Antonio, J., et al. The effects of high-dose glutamine ingestion on weightlifting performance. Journal of Strength and Conditioning Research 16:157-60, 2002.

23. Candow, D., et al. Effect of glutamine supplementation combined with resistance training in young adults. European Journal of Applied Physiology 86:142-49, 2001.

24. Bednarz, B., et al. L-arginine supplementation prolongs exercise capacity in congestive heart failure. Kardiologia Polska 60:34853, 2004.

25. Cheng, J., et al. L-arginine in the management of cardiovascular disease. Annals Pharmacotherapy 35: 755-64, 2001.

26. Fogelholm, G. M., et al. Low-dose amino acid supplementation: No effects on serum growth hormone and insulin in male weightlifters. International Journal of Sport Nutrition 3: 290-97, 1993.

27. Lambert, M., et al. Failure of commercial oral amino acid supplements to increase serum growth hormone concentrations in male body builders. International Journal of Sport Nutrition 3: 298-305, 1993

28. Suminski, R., et al. Acute effect of amino acid ingestion and resistance exercise on plasma growth hormone concentration in young men. International Journal of Sport Nutrition 7: 48-60, 1997.

29. Chromiak, J., and Antonio, J. Use of amino acids as growth-hormone releasing agents by athletes. Nutrition 18: 657-61, 2002.

30. Sutton, E., et al. Ingestion of tyrosine: Effects on endurance, muscle strength, and anaerobic performance. International Journal of Sport Nutrition and Exercise Metabolism 15:173-85, 2005.

31. Baum, M., and Weiss, M. The influence of a taurine containing drink on cardiac parameters before and after exercise measured by echocardiography. Amino Acids 20:75-82, 2001.

32. Zhang, M., et al. Role of taurine supplementation to prevent exercise-induced oxidative stress in healthy young men. Amino Acids 26:203-207, 2004.

33. Gibala, M. Dietary protein, amino acid supplements, and recovery from exercise. Sports Science Exchange15 (4):1-4, 2002.

34. Tipton, K., and Wolfe, R. Exercise-induced changes in protein metabolism. Acta Physiological Scandinavica 162: 377-87, 1998.

35. Walzem, R., et al. Whey components: Millennia of evolution create functionalities for mammalian nutrition. What we know and what we may be overlooking. Critical Reviews in Food Science and Nutrition 42: 353-75, 2002.

36. Brinkworth, G., et al. Oral bovine colostrum supplementation enhances buffer capacity, but not rowing performance in elite female rowers. International Journal of Sport Nutrition and Exercise Metabolism 12: 349-63, 2002.

37. Kuipers, H., et al. Effects of oral bovine colostrum supplementation on serum insulin-like growth factor-I levels. Nutrition 18: 566-7, 2002.

38. Hofman, Z., et al. The effect of bovine colostrum supplementation on exercise performance in elite field hockey players. International Journal of Sport Nutrition and Exercise Metabolism 12:461-69, 2002.

39. Brinkworth, G., et al. Effect of bovine colostrum supplementation on the composition of resistance trained and untrained limbs in healthy young men. European Journal of Applied Physiology 91:53-60, 2004.

40. Tipton, K., and Wolfe, R. Protein and amino acids for athletes. Journal of Sports Sciences 22:65-79, 2004.

41. Poortmans, J., and Dellalieux, O. Do regular high protein diets have potential health risks on kidney function in athletes? International Journal of Sport Nutrition and Exercise Metabolism 10: 28-38, 2000.

42. Tipton, K., and Wolfe, R. Exercise, protein metabolism, and muscle growth. International Journal of Sport Nutrition and Exercise Metabolism 11:109-32, 2001. 